\title{
METHODOLOGY FOR THE ORGANIZATION AND DESIGN OF DISPATCH SERVICES
}

\author{
Kulpunai A. Adylbekova \\ Kyrgyz State Technical University, Bishkek \\ Mohammed Omar Ahmed Abdulwasea \\ Sana'a University, Sana, Republic of Yemen
}

DOI: 10.36724/2664-066X-2020-6-3-2-4

\begin{abstract}
Security and stable socio-economic development of any country depends on the effectiveness of the mechanism for prompt response to emerging threats. Complex of software and hardware "System 112" is designed for automated processing of emergency calls to a single number "112". The design of unified dispatch services (UDS) allows to apply theoretical knowledge to solve urgent practical problems. It is proposed to design the UDS service for mastering the competencies of the educational standard in two stages. Namely, to make preliminary planning and preliminary estimation of the amount of equipment required to deploy the service. Further, the performance indicators of the UDS are evaluated, taking into account the possibility of routing part of the traffic to the dedicated emergency services.
\end{abstract}

KEYWORDS: Unified dispatching service, emergency services, call service center, prompt response, special software.

\section{INTRODUCTION}

Concern for the life and health of citizens, the safety of property, ensuring personal and public safety, as well as the need to counter man-made, natural threats and acts of terrorism require the development of a mechanism for a rapid response to threats. As a single emergency call number in the Russian Federation, 112 was assigned, which combined four emergency services (fire brigade, police, ambulance and gas network emergency service) and two new services (Anti-terror and emergency response service). 


\section{Introduction}

System 112 - multifunctional information and communication geographically distributed automated information and control system created within the boundaries of the subject $[1,2,3,4]$.

The block diagram of system 112 is shown in Figure 1.

It consists of different subsystems providing functions such as:

- receiving and processing calls;

- da abase;

- decision support;

- geo nformation subsystem;

- onitoring;

- raining;

- technological support;

- fixed communication, data transmission and radio communication;

- information security subsystem.

Analysis of the deployment and operation of System 112 in the pilot regions showed that the most important in terms of organizing information interaction of departmental dispatch services is the subsystem for receiving and processing calls (messages about incidents) coming to the unified dispatch service. The specified subsystem includes a call service center (CSC). The subsystem for receiving and processing calls, or the so-called dispatch subsystem (DS), is a control subsystem in the automated system (AS) of the Unified Dispatch Service (UDS).
Currently, several software and hardware options for building this subsystem are possible from the point of view of organizing interagency cooperation. Let's consider two of the most common options.

Option 1. The emergency services DDS $(01,02,03)$ already have their own departmental dispatch systems. Special software (SS) for call processing should be created for the call processing center (CPC) based on the UDDS and for the DS of the emergency operational service, geographically corresponding to the UDS. In practice, this can be implemented by placing a remote client workstation in the DS with the open source software for the call center of the UDS in addition to the departmental AWP for receiving messages about incidents or the development of a special integration software module to ensure information interaction of the open source call center of the call center with the special software for the emergency services.

Option 2. Development of unified automated system of operational dispatch control in each of tDDS separately and as a whole. A unified system should ensure the principle of entering data through one application only once, data should be available for other applications without copying it. All actions of the operator (dispatcher) in the system should be automatically recorded in real time with saving information by whom this action was carried out and when, so that the incident and actions of emergency services personnel on it could be fully restored, including for training goals.

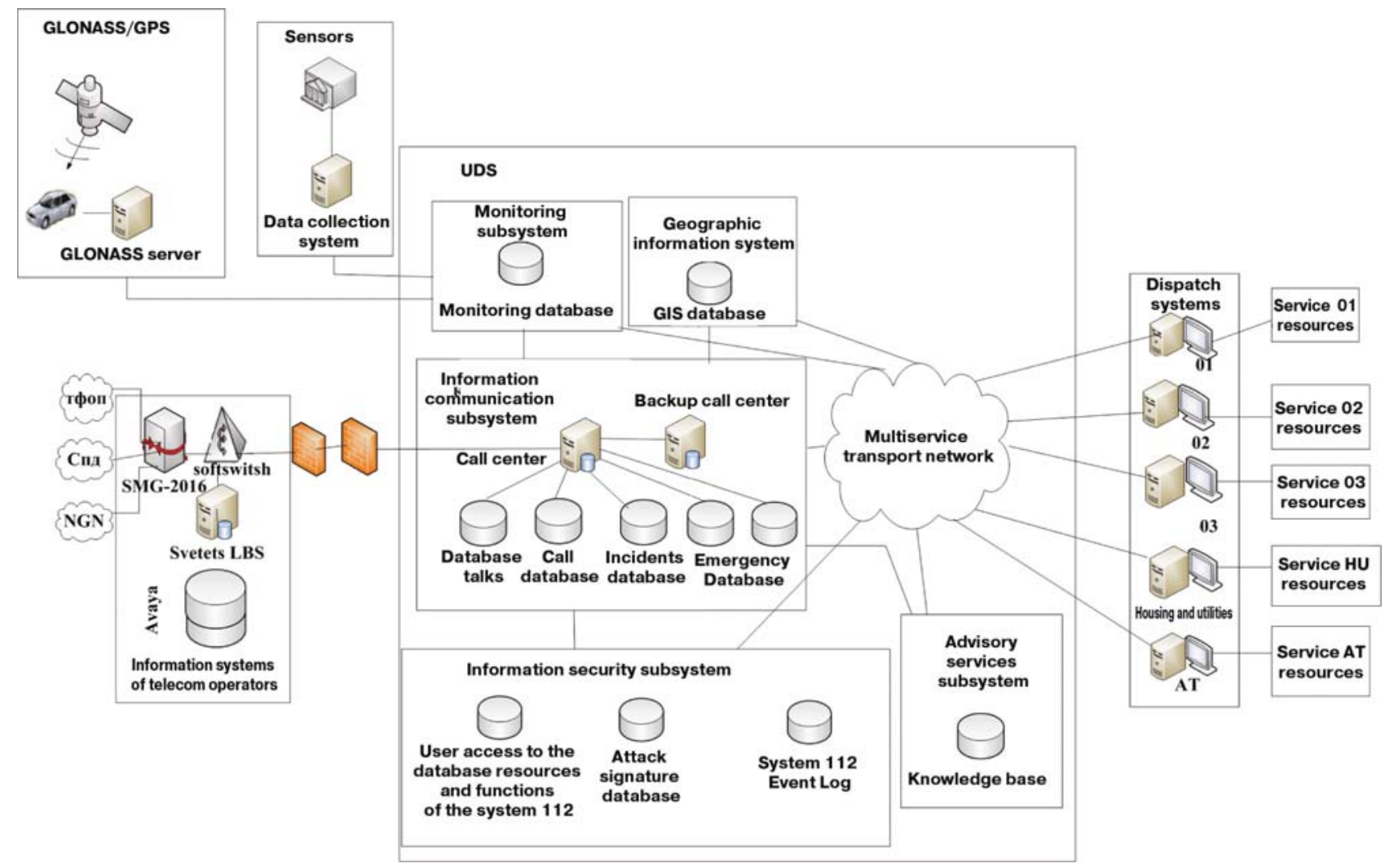

Figure 1. Block diagram of system-112 
Each option has the right to exist and can be implemented in a particular subject. The second option is more preferable, which is more complicated at the initial stage, but later, when the system is replicated in the regions, the implementation effect levels out the problems that arose during development.

As an example of open source software development, we present data for specialized software based on "ISTOK-CM". This is a unique system of instant information exchange and group work, response services of different departmental affiliations, capable of reaching hundreds of organizations - participants involved in emergency and rescue operations and providing prompt decision-making based on the latest reports. The introduction of a standard solution based on the open source software "ISTOK-SM" in UDS and DS of emergency response allows making decisions faster, as it accelerates the collection, processing and distribution of information in all interaction services at all levels.

The Call Service Center (CSC) is an important component of the "System 112", which ensures the reception of calls from the population of the administrative center of the subject and their transfer for service to interacting EDDS, centralized data storage, interaction with the regional navigation and information center of the emergency response system (ERA-GLONASS).

Such call center functions can be implemented on the basis of Avaya Aura Communication Manager (ACM), which, along with calls from landline and mobile telephones, can handle e-mail messages, SMS (which may be more convenient for people with disabilities) and video calls. In the case of an interrupted call, the connection can be automatically restored (Call Back Assist). The development strategy of the solution provides for its integration with applications for smartphones, tablets and with social networks as alternative channels of interaction.

Another current Avaya development is the Avaya Notification Solution (ANS). It is able to provide notification of large groups of the population about an emergency situation through all channels: voice communication (city telephone network, wireless, departmental networks), SMS, e-mail, chats, IP phones, speakers, loudspeakers, text messages on the display phone.

This solution is scalable, highly reliable and can work with equipment from other manufacturers. ANS is currently being implemented, for example, in Netherlands.

To ensure the reliability of the system, a backup call center (BCC) is installed. When the call center fails in the system, the transition to the radio call center is performed in stages $[5,6]$ :

- at he first stage, the call processing is carried out by the duty shift and employees of the BCC admitted to duty, including from among the trainees;

- at the second stage, the staff on duty of the call centers arriving for reinforcement are connected to call processing.

Let us also consider the possibilities of ECSS-10 - a complex solution for building an emergency call service center "System 112" based on the ECSS-10 Softswitch. The hardware and software complex is a combination of software and hardware components developed in Russia, providing a high level of reliability and protection from the presence of "bugs" in the program code.

It is designed to unify the interface of interaction between telecom operators and the call service center of System 112. Telecom operators can connect to UOVEOS using E1 streams (OKS-7) and VoIP protocols SIP, SIP-T, SIP-I. The SIP protocol is used as the interface for interaction between UOVEOS and the System 112 call center.

The ECSS-10 UO-VEOS hardware and software complex consists of the following components:

- program switch ECSS-10 Softswitch;

- trunk gateways SMG-1016M and SMG-2016;

- SBC-1000 and SBC-2000 session border controllers;

- location server TSMN Svetets LBS;

- unified management and monitoring system Eltex EMS.

The general scheme of the "System 112" operation is shown in Figure 2, as an example of a solution by Ericsson Coordcom. The number of dispatchers is variable, as is number of connected response services.

The process of operation of System 112 will be organized in the following way: a message about an incident is received at the call service center 112, or at the DS of one of the emergency services included in System 112. Further, in an automated mode, the operator enters the main characteristics of the incident into the database, transfers them to the DS for the intended purpose, monitors the response to the incident, analyzes and enters the information obtained as a result of the response into the database, if necessary clarifies and corrects the actions of involved DS and informs the interacting DS about operational situation, measures taken and implemented. During and after the end of measures for emergency response to a received call or message, the DS must enter information on the measures taken into the System 112 database.

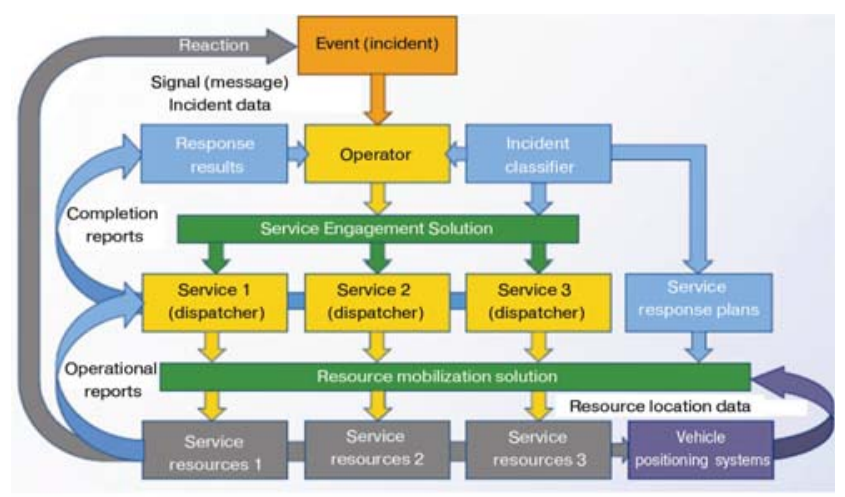

Figure 2. General scheme of emergency response

\section{References}

1. Order of the Government of the Russian Federation of August 25, 2008 No. 1240-r on the approval of the Concept for the creation of System-112.

2. GOST R22. 7.01-99 UDS. Basic provisions.

3. https://www.osp.ru/lan/2012/10/13017656/ (25.11.2019).

4. http://www.myshared.ru/slide/395795/ (25.11.2019).

5. http://www.secuteck.ru/articles2/firesec/sistema-112-otpotrebnostey- k- realizatsii/ (23.11.2019).

6. https://eltexsl.ru/uoveos-solutions/ (25.11.2019).

7. https://nag.ru/articles/article/21574/u-sistem-spaseniya-slojnoebuduschee.html (20.11.2019). 